\title{
ADESÃO AOS MITOS DA CONJUGALIDADE POR HOMENS E MULHERES: ASSOCIAÇÕES COM QUALIDADE E CONFLITO CONJUGAL
}

Angélica Paula Neumann

Universidade Regional Integrada do Alto Uruguai e das Missões - URI - Campus de Erechim

Denise Falcke

Programa de Pós-Graduação em Psicologia da Universidade do Vale do Rio dos Sinos (UNISINOS)

Clarisse Pereira Mosmann

Programa de Pós-Graduação em Psicologia da Universidade do Vale do Rio dos Sinos (UNISINOS)

Adriana Wagner

Programa de Pós-Graduação em Psicologia da Universidade Federal do Rio Grande do Sul

\begin{abstract}
Resumo
O objetivo deste estudo foi identificar a adesão de homens e mulheres a mitos conjugais, comparando os sexos e verificando associações com variáveis sociodemográficas, qualidade conjugal e estilos de resolução de conflitos. Participaram 65 casais heterossexuais, casados oficialmente $(45,3 \%)$, em união estável $(35,9 \%)$ ou namorando $(18,5 \%)$. Os instrumentos utilizados foram: questionário sociodemográfico, questionário de mitos conjugais, Inventário de Estado Conjugal de Golombok e Rust e Inventário de Estilos de Resolução de Conflitos. Os resultados revelaram que houve adesão alta ou moderada aos mitos conjugais. Na amostra masculina, maior adesão foi associada à percepção de maior qualidade conjugal, maior uso de estratégia de submissão e menor envolvimento no conflito. Na amostra feminina, a adesão aos mitos correlacionou-se positivamente com as estratégias de resolução positiva dos conflitos e submissão e negativamente com o envolvimento no conflito. Discute-se a necessidade de considerar a adesão aos mitos da conjugalidade nas intervenções com casais.
\end{abstract}

Palavras-chave: mitos conjugais; sexo; qualidade conjugal; conflito conjugal.

\section{ADHESION TO THE MYTHS OF CONJUGALITY BY MEN AND WOMEN: ASSOCIATIONS WITH MARITAL QUALITY AND CONFLICT}

\begin{abstract}
The purpose of this study was to identify the adhesion of men and women to conjugal myths, comparing the sexes and checking associations with sociodemographic variables, marital quality and style of conflict resolution strategies. Participated 65 heterosexual couples, officially married $(45.3 \%)$, in a stable union $(35.9 \%)$ or dating $(18.5 \%)$. The instruments used were: sociodemographic questionnaire, questionnaire of conjugal myths, Golombok and Rust Inventory of Martital State e Conflict Resolution Style Inventory. The results revealed that there was high or moderate adhesion to the conjugal myths. For men, greater adherence was associated with perceived higher marital quality, greater use of submission strategy and less involvement in conflict. For women, adherence to myths was positively correlated with the strategies of positive resolution of conflicts and submission and negatively with involvement in the conflict. It is discussed the need to consider adherence to the myths of conjugality in interventions with couples.
\end{abstract}

Keywords: conjugal myths; gender; conjugal quality; conjugal conflict. 


\title{
ADHESIÓN A LOS MITOS DE LA CONJUGALIDAD POR HOMBRES Y MUJERES: ASOCIACIONES CON CALIDAD Y CONFLICTO CONJUGAL
}

\begin{abstract}
Resumen
Este estudio analizó la adhesión de hombres y mujeres a los mitos conyugales, haciendo comparaciones entre los sexos y verificando las asociaciones con las variables sociodemográficas, la calidad conyugal y el estilo de resolución de conflictos. Participaron 65 parejas heterosexuales, en matrimonios oficiales $(45,3 \%)$, unión de hecho $(35,9 \%)$ y novios $(18,5 \%)$. Los instrumentos utilizados fueron: cuestionario sociodemográfico y de mitos conyugales, Golombok and Rust Inventory of Martital State e Conflict Resolution Style Inventory. Hubo adhesión alta o moderada a los mitos conyugales. Para los hombres, la mayor adhesión se presentó asociada a la percepción de más calidad conyugal y uso de estrategia de sumisión y menos implicación en el conflicto. Para las mujeres, la adhesión a los mitos se correlacionó positivamente con las estrategias de resolución positiva de los conflictos y la sumisión; y negativamente con la participación en el conflicto. Se discute la necesidad de considerar la adhesión a los mitos conyugales en las intervenciones con parejas.
\end{abstract}

Palabras clave: mitos conyugales; género; calidad conyugal; conflicto conyugal.

\section{INTRODUÇÃO}

As expectativas que as pessoas possuem sobre o seu relacionamento conjugal impactam na avaliação que fazem dele ao longo do tempo. É comum encontrar pessoas insatisfeitas por considerar que o casamento não é aquilo que esperavam. Estudos mostram que, ao se casar, a maior parte dos cônjuges espera que os sentimentos que nutrem pelos seus parceiros permaneçam iguais ou melhorem com o passar dos anos, ignorando a tendência natural de diminuição da satisfação conjugal em determinados períodos da vida a dois (Lavner, Karney, \& Bradbury, 2013), o que evidencia expectativas irrealistas sobre a conjugalidade.

Os mitos conjugais podem influenciar nessas expectativas. Com base em uma revisão de diferentes autores, Andolfi (2018) define os mitos como padrões por meio dos quais a realidade é interpretada, nos quais coexistem alguns elementos reais e outros fantasiosos. Para o autor, os mitos se originam nas histórias contadas pela família de origem e reproduzidas nas sucessivas gerações. Nesse sentido, de acordo com Falcke e Wagner (2005), os mitos podem ser compreendidos como maneiras de explicar diferentes fenômenos da vida, sendo formulados com base em um conjunto de crenças que influenciam nas regras e nos comportamentos que os membros de uma família devem estabelecer entre si e com o mundo exterior. Tais mitos são transmitidos na comunicação pais-filhos (Jackl, 2016) e também reforçados pela cultura e pela mídia (Kramer \& Moore, 2001; Galloway, Engstrom, \& Emmers-Sommer, 2015). Isso porque todos os indivíduos nascem em um contexto no qual existem concepções sobre como deve ser o casamento, além de conviverem com modelos de relacionamentos, sejam de pais, cuidadores, familiares, amigos ou casais, que circulam em filmes e novelas, por exemplo.

Ainda que se façam presentes em todos os relacionamentos (Andolfi \& Angelo, 1989), os mitos conjugais podem contribuir para a manutenção de 
expectativas irreais sobre os relacionamentos. Ainda na década de 1980, Lazarus (1992) listou 24 mitos que carregam ideias irrealistas sobre a conjugalidade. Mitos como "se há amor e compromisso, os casais conseguem enfrentar todas as dificuldades" e "os problemas do início do casamento tendem a melhorar ao longo do tempo" contemplam esperanças idealizadas e impossíveis de serem encontradas na realidade da vida a dois (Wagner \& Mosmann, 2012).

Um ponto que contribui para esse descompasso entre o esperado e a realidade é a pouca clareza que os cônjuges possuem sobre suas expectativas. Nesta perspectiva, um estudo qualitativo realizado com cinco casais portugueses com dois anos de casamento investigou seu período de namoro e revelou que os membros do casal não conversavam abertamente sobre suas expectativas para o matrimônio (Fonseca \& Duarte, 2014). Nessa mesma direção, um estudo qualitativo realizado no Brasil com nove cônjuges em casamentos de longa duração buscou conhecer suas percepções sobre os relacionamentos conjugais na atualidade e revelou um discurso permeado por juízos de valor acerca do que seria esperado de uma relação satisfatória, as quais, embora não tenham sido o foco do estudo, desvelaram uma grande adesão aos mitos conjugais, ainda nos dias atuais (Costa \& Mosmann, 2015).

A literatura apresenta controvérsias sobre o impacto da adesão a mitos conjugais na qualidade do relacionamento. A qualidade conjugal é um construto difícil de ser mensurado, mas pode ser definida como a avaliação subjetiva que os cônjuges fazem do próprio relacionamento (Mosmann, Wagner, \& FéresCarneiro, 2006). Envolve aspectos como satisfação, comunicação, interesses compartilhados, confiança e respeito (Rust et al., 1988). Embora a maioria dos estudos evidencie que a adesão a mitos conjugais pode comprometer a qualidade do relacionamento (Caldwell \& Woolley, 2008; Ercegovac \& Bubić, 2016; Fowler, 2000), um estudo americano feito com mulheres indica que ela pode predizer experiências conjugais positivas (Casad, Salazar, \& Macina, 2015). Investigando 99 mulheres em relacionamentos conjugais heterossexuais na etapa do noivado, nos Estados Unidos, Casad et al. (2015) demonstraram que as mulheres que rejeitaram os mitos conjugais e endossaram o sexismo benevolente apresentaram mais padrões interacionais negativos, incluindo menor confiança e satisfação nos seus relacionamentos.

Isso pode ser explicado devido a que possuir uma expectativa otimista de ter um relacionamento estável, prazeroso e de qualidade, tende a ser característica de pessoas que conseguem manejar de forma mais construtiva os desentendimentos inerentes à vida a dois. Contudo, esperar que o relacionamento não passe por experiências difíceis, ou passe por poucas situações adversas, caracteriza aquelas pessoas que encontram maior dificuldade de resolver os conflitos conjugais, especialmente os mais difíceis (Neff \& Geers, 2013). Na mesma direção, pesquisa realizada com 120 casais heterossexuais israelenses em casamentos de longa duração demonstrou que ter expectativas 
apropriadas a respeito dos deveres do parceiro(a) para consigo se relaciona com bons níveis de satisfação conjugal, já que as expectativas também têm como papel indicar o tipo de investimento feito no relacionamento (George-Levi, Vilchinsky, Tolmacz, \& Liberman, 2014).

Sendo assim, pode-se pensar que o principal problema das expectativas sobre os relacionamentos conjugais ocorre quando elas não são realistas. Um estudo realizado com 384 pessoas casadas na Turquia revelou que a insatisfação conjugal esteve positivamente associada com a dependência emocional, a percepção de rejeição interpessoal e crenças irrealistas sobre o relacionamento (Kemer, Yıldız, \& Bulgan, 2016). Tais evidências demonstram a importância de esclarecer aos casais acerca dos desafios que fazem parte da vida a dois, o que pode ser difícil uma vez que inclusive terapeutas de casal e família tendem a aderir a mitos relacionados à conjugalidade (Caldwell \& Woolley, 2008). Esta tarefa, então, representa uma função normalizadora, ou seja, demonstra a necessidade de compreender que os mitos conjugais, assim como as dificuldades e conflitos, são inerentes à vida conjunta (Costa, Cenci, \& Mosmann, 2016; Delatorre, Scheeren, \& Wagner, 2017; Mosmann \& Falcke, 2011).

Revisão sistemática da literatura sobre conflitos conjugais e estratégias de resolução, desenvolvida por Costa et al. (2016), identificou que as estratégias de resolução descritas na literatura se apresentam como construtivas quando há uma postura flexível e tolerante dos cônjuges diante dos problemas conjugais e limitações do parceiro, assim como uma percepção otimista sobre os conflitos e sobre a responsabilidade mútua em resolvê-los. Por outro lado, as estratégias destrutivas estão relacionadas aos comportamentos individualizados frente aos problemas e às dificuldades em flexibilizar pensamentos e se conectar com o que há de positivo na relação e no parceiro.

Dessa forma, os mitos conjugais podem contribuir para a dificuldade em flexibilizar os pensamentos sobre o funcionamento conjugal, promovendo a utilização de estratégias disfuncionais, tais como o envolvimento no conflito, a submissão ou o afastamento do conflito. O envolvimento no conflito refere-se a ataques ao parceiro, insultos e perda do controle. O afastamento corresponde à recusa em discutir o conflito em questão e ao silêncio prolongado. Por sua vez, a submissão indica condescendência, desistência em defender seu ponto de vista e aceitação passiva da opinião do outro (Kurdek, 1994).

Diferenças de gênero costumam existir na utilização dessas estratégias, sendo característico um padrão de demanda por parte das mulheres e recuo por parte dos homens (Delatorre et al., 2017). Também já foram observadas diferenças de gênero na adesão aos mitos conjugais (Honeycutt, 1991), indicando que os homens demonstraram maior concordância com os mitos apresentados. Todavia, não foram localizados estudos que avaliassem a interação entre adesão aos mitos conjugais e características de conflito e qualidade conjugal. 
Sendo assim, o objetivo deste estudo foi identificar os níveis de adesão de homens e mulheres a quatro mitos conjugais, avaliando se existe diferença conforme o sexo. Além disso, buscou-se verificar associações entre a adesão aos mitos com variáveis sociodemográficas, qualidade conjugal e estilo de resolução de conflitos. Dentre os mitos investigados no presente estudo, três foram propostos por Lazarus (1992), sendo eles "quem ama de verdade adivinha o que o outro pensa e sente", "marido e mulher devem ser melhores amigos" e "o casamento é a fonte de toda a felicidade" e um foi proposto no programa Viver a dois: Compartilhando este desafio, sendo ele "um casal saudável é aquele que não possui conflitos" (Wagner et al., 2015). Entende-se que uma melhor compreensão sobre esse fenômeno pode contribuir para futuras intervenções com foco na prevenção de conflitos conjugais e promoção da qualidade nos relacionamentos.

\section{MÉTODO}

\section{Participantes}

A amostra do estudo foi composta por 65 casais heterossexuais. A maior parte coabitava e era casada $(45,3 \%, n=29)$ ou vivia em união estável $(35,9 \%$, $\mathrm{n}=23$ ), enquanto 12 casais namoravam há mais de seis meses e não coabitavam no momento da coleta de dados (18,5\%). Destes casais, $50,8 \%$ residiam em metrópole ou na região metropolitana de um estado do sul do país $(n=33), 20 \%$ residiam em cidades do interior do estado com mais de 100 mil habitantes ( $n=13$ ) e $29,2 \%$ residiam em cidades do interior do estado com menos de 20 mil habitantes $(n=19)$. O tempo total de relacionamento foi de, em média, 12,3 anos ( $D P=11,30)$, e de coabitação para aqueles que residiam juntos de, em média, 11,9 anos (DP = 10,60).

Os homens tinham 37 anos de idade em média ( $D P=11,26$ ) e as mulheres 35,6 anos ( $D P=11,12)$. Cerca de metade da amostra masculina possuía Ensino Superior Completo ou Incompleto, e aproximadamente um terço concluiu o Ensino Médio. Para as mulheres, cerca de $70 \%$ possuía Ensino Superior Completo ou Incompleto, e aproximadamente um quinto estudou até o Ensino Médio. A maioria dos homens $(78,5 \%, n=51)$ e das mulheres $(81,5 \%, n$ = 53) exerciam atividade remunerada, com vencimentos pessoais de até três salários mínimos para a maior parte dos participantes. Dentre os homens, 29,2\% ( $n=19)$ já viveu um relacionamento de coabitação anterior, assim como $26,2 \%$ ( $n=17)$ das mulheres. A maior parte dos homens $(63,1 \%, n=41)$ e das mulheres $(66,2 \%, n=43)$ tinham filhos. A caracterização dos participantes pode ser visualizada na Tabela 1. 
Tabela 1.

Caracterização dos participantes

\begin{tabular}{|c|c|c|c|c|}
\hline & \multicolumn{2}{|c|}{ Homens } & \multicolumn{2}{|c|}{ Mulheres } \\
\hline & $\%$ & $n$ & $\%$ & $n$ \\
\hline \multicolumn{5}{|l|}{ Situação conjugal } \\
\hline Casados & 45,3 & 29 & 45,3 & 29 \\
\hline União estável & 35,9 & 23 & 35,9 & 23 \\
\hline Namoro & 18,5 & 12 & 18,5 & 12 \\
\hline \multicolumn{5}{|l|}{ Cidade em que residiam } \\
\hline Metrópole/região metropolitana & 50,8 & 33 & 50,8 & 33 \\
\hline Interior > 100 mil habitantes & 20 & 13 & 20 & 13 \\
\hline Interior $<20$ mil habitantes & 29,2 & 19 & 29,2 & 19 \\
\hline \multicolumn{5}{|l|}{ Escolaridade } \\
\hline Ens. Fundamental Completo ou Incompleto & 13,8 & 9 & 10,8 & 7 \\
\hline Ens. Médio Completo ou Incompleto & 30,8 & 20 & 18,5 & 12 \\
\hline Ens. Superior Completo ou Incompleto & 55,4 & 36 & 70,8 & 46 \\
\hline \multicolumn{5}{|l|}{ Exerce atividade remunerada } \\
\hline Sim & 78,5 & 51 & 81,5 & 53 \\
\hline Não & 21,5 & 14 & 18,5 & 12 \\
\hline \multicolumn{5}{|l|}{ Renda pessoal em Salários Mínimos (SM) } \\
\hline Sem renda & 24,6 & 16 & 16,9 & 11 \\
\hline Até três SM & 43,1 & 28 & 52,3 & 34 \\
\hline Mais do que três, até seis SM & 16,9 & 11 & 12,3 & 8 \\
\hline Mais do que seis SM & 15,4 & 10 & 18,4 & 12 \\
\hline \multicolumn{5}{|l|}{ Relacionamento de coabitação anterior } \\
\hline Sim & 29,2 & 19 & 26,2 & 17 \\
\hline Não & 70,8 & 46 & 72,3 & 47 \\
\hline \multicolumn{5}{|l|}{ Tem filhos } \\
\hline Sim & 63,1 & 41 & 66,2 & 43 \\
\hline \multirow[t]{2}{*}{ Não } & 36,9 & 24 & 33,8 & 22 \\
\hline & $M$ & $D P$ & $M$ & $D P$ \\
\hline Tempo total de relacionamento & 12,3 & 11,3 & 12,3 & 11,3 \\
\hline Tempo de coabitação & 11,9 & 10,6 & 11,9 & 10,6 \\
\hline Idade & 37 & 11,26 & 35,6 & 11,12 \\
\hline
\end{tabular}

Instrumentos

Os 65 homens e 65 mulheres que integraram a amostra responderam, individualmente, ao seguinte conjunto de instrumentos: 


\section{Questionário sociodemográfico}

Investigou dados a respeito da idade, escolaridade, ocupação, renda, cidade em que residia, tempo de relacionamento, filhos e relações anteriores.

\section{Questionário sobre mitos conjugais}

Desenvolvido por Neumann (2017) a partir dos mitos trabalhados no Programa Psicoeducativo para Casais Viver a dois: Compartilhando este desafio (Wagner et al., 2015) para avaliar a adesão a quatro mitos conjugais em uma escala Likert de 6 pontos, em que $1=$ discordo plenamente e $6=$ concordo plenamente. Os mitos avaliados foram: "quem ama de verdade adivinha o que o outro pensa e sente", "marido e mulher devem ser melhores amigos", "o casamento é a fonte de toda a felicidade" e "um casal saudável é aquele que não possui conflitos".

Inventário de Estado Conjugal de Golombok e Rust (GRIMS; Rust et al., 1988; traduzida e adaptada à língua portuguesa por Falcke, 2003)

Foi utilizado para averiguar a qualidade conjugal dos participantes. Tratase de uma escala composta por 28 itens, os quais são pontuados em uma escala Likert de quatro pontos (discordo fortemente, discordo, concordo e concordo fortemente). Esta escala mede a qualidade do relacionamento conjugal por meio de dimensões que são consideradas importantes para um bom relacionamento, sendo elas: satisfação, comunicação, interesses compartilhados, confiança e respeito. Apesar disso, a pontuação é feita de maneira unidimensional através da soma dos pontos dos 28 itens. Conforme a diretriz de Rust et al. (1988), quanto maiores os escores obtidos, mais severos são os problemas no relacionamento conjugal. Como forma de facilitar a interpretação das correlações, porém, no presente estudo todos os itens tiveram a pontuação invertida de modo que, quanto maiores os escores obtidos, maior a qualidade da relação. O Alfa de Cronbach encontrado no presente estudo foi de 0,86 .

Inventário de Estilos de Resolução de Conflitos (CRSI; Kurdek, 1994, traduzido e adaptado para o português brasileiro por Scheeren et al., 2014, e validado por Delatorre et al., 2017)

Foi empregado para verificar os estilos de resolução de conflitos utilizados pelos participantes. O CRSI investiga quatro estilos de resolução de conflitos conjugais por meio de 16 itens, medidos em uma escala Likert de cinco pontos ( 1 = nunca e $5=$ sempre). 0 primeiro estilo é a Resolução positiva dos problemas, caracterizada pelo uso de negociações e busca de compromisso. $O$ segundo é o Envolvimento no conflito, que se distingue pelo uso de ataques pessoais e perda de controle durante uma discussão. O Afastamento ocorre quando um dos membros do casal se recusa a continuar discutindo um assunto. A Submissão, por sua vez, é demarcada pela desistência do indivíduo em 
defender a sua posição, adotando uma postura de obediência. Os estilos foram computados conforme proposto por Delatorre et al. (2017). Os valores do Alfa de Cronbach encontrados neste estudo para as subescalas foram 0,72 para Resolução positiva, 0,79 para Envolvimento no conflito, 0,63 para Afastamento e 0,50 para Submissão.

\section{Procedimentos}

Este estudo integra uma investigação maior, que foi aprovada pelo Comitê de Ética em Pesquisa da Universidade Federal do Rio Grande do Sul (Parecer no. 1.171.899). Os participantes são casais que responderam a um convite para participar do Programa Psicoeducativo para Casais "Viver a dois: Compartilhando este Desafio" (Wagner et al., 2015), realizado em cinco cidades do Sul do Brasil, nos anos de 2015 e 2016. O programa foi conduzido por profissionais da psicologia, saúde, educação e assistência social, vinculados a Secretarias de Saúde, Centros de Referência e de Referência Especializada em Assistência Social (CRAS e CREAS), Centros de Formação em Terapia de Família e Universidades. A divulgação do programa para recrutamento dos casais ocorreu por meio de redes sociais, mídia, folders, cartazes e convite pessoal. Os interessados se inscreveram para participar de uma reunião informativa, na qual foram explicados os procedimentos do programa e da pesquisa. Aqueles que concordaram com os procedimentos expostos assinaram o Termo de Consentimento Livre e Esclarecido e responderam ao questionário no dia desta reunião informativa. Este estudo, assim, utiliza os dados coletados neste momento da investigação, antes de os casais iniciarem as oficinas do Programa.

Para a análise dos resultados, inicialmente, procedeu-se avaliação da normalidade dos dados a fim de verificar os pressupostos necessários para a realização de análises paramétricas. Uma vez que parte das variáveis não apresentou distribuição normal, optou-se pela utilização de estatísticas não paramétricas. Desta forma, para identificar os níveis de adesão de homens e mulheres aos mitos conjugais, realizaram-se análises descritivas de medianas e intervalos interquartílicos.

Para verificar a existência de diferença no nível de adesão aos mitos entre homens e mulheres, utilizou-se o teste $U$ de Mann-Whitney. $O$ tamanho do efeito da diferença foi investigado por meio do estatístico $d$ de Cohen, considerando os valores $d$ entre 0,1 e 0,4 como representativos de um efeito pequeno, valores $d$ entre 0,5 e 0,7 de um efeito intermediário e valores $d$ acima de 0,8 de um tamanho de efeito grande (Cohen, 1988).

Por fim, para examinar a existência de relações entre a adesão aos mitos com variáveis sociodemográcas, qualidade conjugal e estilos de resolução de conflitos, utilizou-se a análise de correlações $p$ de Spearman. Considerou-se que valores positivos e negativos entre 0,1 e 0,3 indicam correlações fracas, entre 
0,4 e 0,6 indicam correlações moderadas, entre 0,7 e 0,9 indicam correlações fortes e 1 indica correlação perfeita (Dancey \& Reidy, 2006).

\section{RESULTADOS}

Em geral, os dados evidenciaram níveis de adesão moderados a altos, tanto para homens quanto para mulheres, em três dos quatro mitos investigados. Percebe-se alta adesão ao mito "Marido e mulher devem ser melhores amigos", e níveis moderados de adesão aos mitos "Quem ama de verdade adivinha o que o outro pensa e sente" e "O casamento é a fonte de toda a felicidade". O mito ao qual houve menos adesão foi "Um casal saudável é aquele que não possui conflitos", estando as mulheres ainda mais predispostas a discordar do mesmo, conforme pode ser observado na Tabela 2.

Tabela 2.

Diferença entre homens e mulheres na adesão aos mitos conjugais

$$
\text { Homens Mulheres }
$$

\begin{tabular}{|c|c|c|c|c|c|c|}
\hline \multirow{2}{*}{ Mito } & \multicolumn{2}{|c|}{ encon } & \multicolumn{2}{|c|}{ 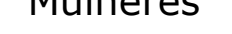 } & \multirow[b]{2}{*}{$\begin{array}{l}U \text { de Mann- } \\
\text { Whitney }\end{array}$} & \multirow[b]{2}{*}{$\begin{array}{l}d \text { de } \\
\text { Cohen }\end{array}$} \\
\hline & Mdn & $I Q R$ & Mdn & $I Q R$ & & \\
\hline $\begin{array}{l}\text { Quem ama de verdade adivinha } \\
\text { o que o outro pensa e sente }\end{array}$ & 4,00 & 2,50 & 3,00 & 2,75 & $1.385,500 *$ & 0,62 \\
\hline $\begin{array}{l}\text { Marido e mulher devem ser } \\
\text { melhores amigos }\end{array}$ & 6,00 & 1,00 & 5,00 & 2,00 & $1.682,500 * * *$ & 0,36 \\
\hline $\begin{array}{l}\text { O casamento é a fonte de toda } \\
\text { a felicidade }\end{array}$ & 4,00 & 3,00 & 3,00 & 2,75 & $1.919,000$ & 0,16 \\
\hline $\begin{array}{l}\text { Um casal saudável é aquele } \\
\text { que não possui conflitos }\end{array}$ & 3,00 & 2,50 & 2,00 & 2,00 & $1.440,500 *$ & 0,57 \\
\hline
\end{tabular}

Percebem-se diferenças estatisticamente significativas entre as respostas de homens e de mulheres em três mitos, sendo que os homens demonstraram maior propensão a acreditar neles. A diferença entre homens e mulheres nos mitos "Quem ama de verdade adivinha o que o outro pensa e sente" e "Um casal saudável é aquele que não possui conflitos" obteve tamanho de efeito intermediário, enquanto a diferença na crença ao mito "Marido e mulher devem ser melhores amigos" obteve tamanho de efeito pequeno. Não houve diferenças estatisticamente significativas entre homens e mulheres em relação ao mito "O casamento é a fonte de toda a felicidade" (Tabela 2).

A fim de investigar se existem relações entre a adesão aos mitos com variáveis sociodemográficas, qualidade conjugal e estilo de resolução de conflitos, utilizou-se a análise de correlação de Spearman. Os resultados podem ser observados na Tabela 3. 
Quando se analisam apenas os mitos, pode-se perceber a existência de correlações múltiplas entre eles. Evidencia-se que as crenças nos três primeiros mitos estão positivamente correlacionadas entre si, tanto para homens quanto para mulheres, sendo algumas destas correlações consideradas fracas e outras moderadas. O mito "Um casal saudável é aquele que não possui conflitos" se distingue dos demais ao não se correlacionar com todos os outros mitos. Para os homens, essa ideia se correlaciona com os mitos "Quem ama de verdade adivinha o que o outro pensa e sente" e "O casamento é a fonte de toda a felicidade". Já para as mulheres, se relaciona apenas com o mito "O casamento é a fonte de toda a felicidade".

No que diz respeito às variáveis sociodemográficas, para as mulheres, a adesão ao mito "Quem ama de verdade adivinha o que o outro pensa e sente" se relaciona positivamente com a idade e com o tempo total de relacionamento. Para os homens, a variável renda correlacionou-se negativamente com alguns mitos. Quanto maior a renda, menor a crença nas ideias "Marido e mulher devem ser melhores amigos" e "O casamento é a fonte de toda a felicidade".

Tabela 3

Correlações entre mitos conjugais e variáveis sociodemográficas, qualidade conjugal e estilos de resolução de conflitos, para homens e mulheres

\begin{tabular}{|c|c|c|c|c|c|c|c|c|}
\hline & \multicolumn{4}{|c|}{ Homens } & \multicolumn{4}{|c|}{ Mulheres } \\
\hline & Mito 1 & Mito 2 & Mito 3 & Mito 4 & Mito 1 & $\begin{array}{c}\text { Mito } \\
2\end{array}$ & $\begin{array}{c}\text { Mito } \\
3\end{array}$ & $\begin{array}{c}\text { Mito } \\
4\end{array}$ \\
\hline Mito 1 & 1 &, $331^{* *}$ &, $509^{* *}$ & $359^{* *}$ & 1 &, $318^{*}$ & $363^{*}$ & 159 \\
\hline Mito 2 &, $331^{* *}$ & 1 &, $366^{* *}$ & 101 &, $318^{* *}$ & 1 & $475^{*}$ & , 126 \\
\hline Mito 3 &, $509^{* *}$ &, $366^{* *}$ & 1 &, $505^{* *}$ &, $363^{* *}$ &, $475^{*}$ & 1 &, $395^{*}$ \\
\hline Mito 4 &, $359^{* *}$ & 101 &, $505^{* *}$ & 1 & 159 & , 126 &, $395^{*}$ & 1 \\
\hline Idade & ,020 &,- 241 &,- 111 &,- 117 &, $303^{*}$ & ,019 &,- 011 & ,009 \\
\hline $\begin{array}{l}\text { Tempo de } \\
\text { relação (meses) }\end{array}$ & 112 &,- 143 &, 053 &,- 068 &, $397^{* *}$ & 126 & 172 &,- 153 \\
\hline Renda pessoal & 149 &,$- 342^{*}$ &,$- 425^{* *}$ &,- 187 &,- 177 &,- 228 &,- 229 &,- 123 \\
\hline $\begin{array}{l}\text { Qualidade } \\
\text { conjugal }\end{array}$ & $281^{*}$ &, 162 &, $312^{*}$ &,- 016 &,- 119 &,- 094 &,- 129 &,- 032 \\
\hline $\begin{array}{l}\text { Resolução } \\
\text { positiva }\end{array}$ &, 155 & ,073 & 127 &,- 075 &, $306^{*}$ &,- 126 &,- 014 & 193 \\
\hline $\begin{array}{l}\text { Envolvimento } \\
\text { no conflito }\end{array}$ &,- 190 &,- 123 &,$- 272^{*}$ &,- 028 &,$- 378^{* *}$ &,- 016 &,- 096 &,- 054 \\
\hline Afastamento &,- 081 & 241 &,- 018 &,- 021 & ,014 & 147 & 182 & 100 \\
\hline Submissão &, 161 & 170 & 143 &, $359^{* *}$ & ,068 & ,223* & ,279* & 142 \\
\hline
\end{tabular}


$* \mathrm{p}<0,05, * * \mathrm{p}<0,01$

Nota: Mito 1. Quem ama de verdade adivinha o que o outro pensa e sente; Mito 2. Marido e mulher devem ser melhores amigos; Mito 3. O casamento é a fonte de toda a felicidade; Mito 4. Um casal saudável é aquele que não possui conflitos.

Já no que se refere à qualidade conjugal, contrariando a hipótese, observou-se correlação positiva, para os homens, entre qualidade conjugal e adesão à crença de que "Quem ama de verdade adivinha o que o outro pensa e sente" e "O casamento é a fonte de toda a felicidade". Para as mulheres, não há correlação entre a qualidade conjugal e os mitos investigados.

Por fim, no tocante aos estilos de resolução de conflitos, para os homens, há correlação negativa entre envolvimento no conflito e adesão ao mito de que "O casamento é a fonte de toda a felicidade", assim como uma correlação positiva entre o estilo de submissão e o mito "Um casal saudável é aquele que não possui conflitos". No caso das mulheres, a adesão ao mito "Quem ama de verdade adivinha o que o outro pensa e sente" se correlacionou positivamente com a resolução positiva de conflitos e negativamente com o envolvimento no conflito. Por fim, o estilo de submissão se correlacionou positivamente com os mitos "Marido e mulher devem ser melhores amigos" e "O casamento é a fonte de toda a felicidade".

\section{DISCUSSÃO}

Os resultados do estudo evidenciam adesão moderada e alta a três dos mitos apresentados, indicando o quanto os indivíduos incorporam as concepções que são transmitidas culturalmente sobre os relacionamentos, conforme já evidenciado na literatura (Kramer \& Moore, 2001; Galloway et al., 2015; Jackl, 2016), mesmo quando elas podem ser consideradas mitos. O único mito ao qual os participantes demonstraram menores níveis de adesão foi "Um casal saudável é aquele que não possui conflitos", possivelmente por ser mais disseminada a noção de que os conflitos são inerentes a todos os relacionamentos (Costa et al., 2016; Delatorre et al., 2017; Mosmann \& Falcke, 2011). Também foi possível constatar maior adesão masculina aos mitos, confirmando estudo prévio (Honeycutt, 1991). Uma possível explicação para esse dado é o fato de as mulheres refletirem e dialogarem mais durante a vida sobre os relacionamentos afetivos, o que favorece que questionem ideias pré-concebidas, enquanto os homens parecem aderir mais diretamente aos mitos que circundam o imaginário social com menor questionamento.

Foram observadas múltiplas correlações entre os mitos, indicando a coerência implícita que existe entre eles no que se refere às ideias de que o casamento é um relacionamento que implica sentimentos de amizade, romantismo e felicidade. Se por um lado essas crenças podem ser protetivas por 
significarem maior nível de investimento afetivo dos cônjuges no relacionamento (George-Levi et al., 2014), por outro, podem se tornar um fator de risco ao expressar necessidades que, provavelmente, o relacionamento conjugal não possa oferecer, especialmente quando se acredita que o casamento é a fonte de toda a felicidade.

Considerando as variáveis sociodemográficas, houve associação, na amostra feminina, entre idade e tempo de relacionamento com o mito de que "Quem ama de verdade, adivinha o que o outro pensa ou sente". Nesse sentido, pode-se pensar que haja uma confusão entre o conhecimento do outro que se adquire com o passar do tempo e a ideia de que esse conhecimento advenha do amor verdadeiro. Também pode corresponder à expectativa de que os sentimentos pelo parceiro melhoram com o passar dos anos (Lavner et al., 2013). No estudo de Costa e Mosmann (2015), os casais apontaram que, com o passar do tempo, existe uma adaptação entre os cônjuges, vinculada ao tempo de união e ao conhecimento do parceiro, que faz com eles acreditem antecipadamente que sabem o que o outro está pensando. Entretanto, a clínica com casais indica justamente o oposto, pois é a partir dessa crença de que um conhece o outro que muitos casais entram em conflito, por não conseguirem manter um diálogo aberto e sim uma troca de suposições acerca do que o outro pensa, as quais, muitas vezes, estão embasadas nesse mito. Quando essa crença prevalece, os cônjuges podem deixar de perguntar um ao outro sobre as suas opiniões por já imaginarem as respostas, perdendo, assim, oportunidades de conexão emocional. Além disso, muitos indivíduos podem se sentir frustrados quando suas intenções não são imediatamente compreendidas pelo parceiro(a), entendendo isso como falta de interesse do outro.

$\mathrm{Na}$ amostra masculina, a renda se associou negativamente aos mitos "Marido e mulher devem ser melhores amigos" e "O casamento é fonte de toda a felicidade". É possível pensar que o aumento na renda abre possibilidades de outras vivências prazerosas, retirando da conjugalidade a responsabilidade pela felicidade e satisfação global de vida. No estudo de Delatorre et al. (2017), os participantes homens com renda mais elevada e os que trabalhavam fora de casa utilizavam com mais frequência a estratégia de afastamento frente aos conflitos, possivelmente por encontrar refúgio às discordâncias maritais em outras atividades externas à relação. Naquela investigação, apesar de os participantes fazerem uso do afastamento, a estratégia mais utilizada ainda foi a resolução positiva. Conjugando tais dados com os resultados do presente estudo, pode-se pensar que se cada um dos membros do casal consegue desenvolver e preservar outras fontes de satisfação, que não sejam exclusivamente vinculadas à conjugalidade, podem se sentir mais fortalecidos e desenvolver formas mais positivas de encaminhar os problemas do relacionamento conjugal, sem ficarem tão ameaçados pela discórdia com o outro. 
A qualidade conjugal só foi associada à adesão aos mitos na amostra masculina. Em relação ao primeiro mito, a princípio, se poderia pensar que um alto índice de adesão poderia sobrecarregar a relação e assim diminuir a qualidade conjugal. Porém, os resultados demonstraram que a maior adesão se relaciona ao aumento da percepção de qualidade no relacionamento, corroborando estudo prévio (Casad et al., 2015) que já indicou que a adesão a mitos pode predizer experiências positivas. É possível que aqueles homens que vivem boa qualidade conjugal possuam maior intimidade e diálogo no seu relacionamento, o que os leva a acreditar que possuem maior facilidade em "adivinhar" o que a parceira quer, assim como percebê-la como sendo um de seus melhores amigos. A adesão ao mito e sua expressão em maior qualidade conjugal também pode estar associada ao compromisso decorrente dessa crença, que leva o sujeito a comportar-se segundo o que acredita e, consequentemente comprovar o mito, conforme já foi indicado em outros estudos (Rizzon, Mosmann, \& Wagner, 2013). Além disso, cabe ressaltar que a análise aqui realizada é individual, essa é a percepção de um membro da díade conjugal acerca de sua vivência de qualidade conjugal, não significa que seja correspondente a sua companheira.

A adesão aos mitos também esteve associada a diferentes estratégias de resolução de conflitos. Para os homens, a crença no mito "O casamento é a fonte de toda a felicidade" associou-se negativamente ao envolvimento no conflito. Pode-se pensar que, quando o homem supervaloriza o relacionamento conjugal como fonte de prazer, ele evita o ataque e o insulto à parceira como forma de preservar a relação. Além disso, maior adesão ao mito "Um casal saudável é aquele que não possui conflitos" relacionou-se ao uso mais frequente da estratégia de submissão, ou seja, os homens parecem considerar que é melhor se submeter aos desejos da parceira a entrar em conflito, visto que o conflito significaria infelicidade. Por sua vez, circularmente, a ideia de que o conflito é algo ruim pode fazer com que se submetam. Essas estratégias masculinas são condizentes à posição de recuo frente aos conflitos conjugais já reconhecida na literatura (Delatorre et al., 2017).

Na amostra feminina, a adesão ao mito "Quem ama de verdade adivinha o que o outro pensa e sente" correlacionou-se positivamente com as estratégias de resolução positiva dos conflitos e negativamente com o envolvimento no conflito. Sendo assim, tem-se a noção da adesão a esse mito como algo protetivo ao relacionamento, propiciando maior negociação nas situações de conflito e menos ataques ao parceiro. Considerando que esse mito se associou também, na amostra feminina, à idade e ao tempo de relacionamento, pode-se novamente refletir sobre a possível confusão entre o conhecimento do outro e a intimidade advindos da convivência com a ideia de poder ler ou adivinhar o pensamento do companheiro. Além disso, a adesão aos mitos "O casamento é a fonte de toda a felicidade" e "Marido e mulher devem ser melhores amigos" associou-se 
positivamente à estratégia de submissão. Nesse caso, pode-se refletir sobre o quanto, quando o casamento é fonte única de satisfação, existe defensivamente a necessidade de evitar o confronto em situação de conflito, recorrendo à submissão. Isso pode ser amplificado quando se percebe o parceiro como o melhor amigo. Desde uma perspectiva de retroalimentação, a dificuldade de se posicionar pode fazer com que se tema o fim do relacionamento, levando à dedicação integral ao mesmo e à perda da individualidade na busca por outras relações e atividades prazerosas fora da relação.

De maneira geral, pode-se considerar que, no presente estudo, a adesão aos mitos não foi comprometedora da qualidade do relacionamento, contrariando a ideia que é recorrente na literatura especializada no tema (Caldwell \& Woolley, 2008; Ercegovac \& Bubić, 2016; Fowler, 2000). Partindo da premissa de Andolfi e Angelo (1989) de que mitos conjugais se fazem presentes em todos os relacionamentos, é possível que o mais prejudicial à conjugalidade seja a existência de expectativas extremamente irrealistas (Lazarus, 1992). Quando os mitos funcionam como uma forma de manter expectativas positivas a respeito da relação (George-Levi et al., 2014, Neff \& Geers, 2013) é possível que não se constituam em fenômeno tão deletério aos relacionamentos, contribuindo com maior satisfação conjugal, conforme aparece na amostra masculina e com estratégias de resolução positiva dos conflitos (no caso das mulheres) e menor envolvimento no conflito (para ambos os sexos). Ressalta-se, contudo, a necessidade de atenção à associação da adesão aos mitos com estratégias de submissão, pois essa refere-se à aceitação passiva do desejo/opinião do outro, sem defesa do próprio ponto de vista (Kurdek, 1994), o que pode gerar acúmulo de frustrações e, com o tempo, acúmulo de tensões.

\section{CONSIDERAÇÕES FINAIS}

O objetivo deste estudo foi identificar os níveis de adesão de homens e mulheres a quatro mitos conjugais, avaliando se existem diferenças conforme o sexo. Além disso, buscou-se verificar se existem associações entre a adesão aos mitos com variáveis sociodemográficas, qualidade conjugal e estilos de resolução de conflitos. Avalia-se que os mesmos foram atingidos, fornecendo resultados interessantes acerca de tema pouco investigado no contexto brasileiro.

Destaca-se a adesão de ambos os sexos aos mitos, em alguma medida, e as reverberações na qualidade conjugal percebida pelos homens. Nesse sentido, novas pesquisas poderiam contribuir para uma melhor compreensão dessas diferenças através de delineamentos qualitativos através dos quais os participantes pudessem expressar suas considerações acerca dos mitos a respeito da conjugalidade. Ao utilizar-se o método de autorrelato, foi feita uma proposição aos participantes de quatro mitos que se encontram na literatura, mas não se sabe se haveria outros aos quais os mesmos se sentem mais ou 
menos aderidos. Além disso, considera-se importante compreender se e como a adesão aos mitos muda com o passar dos anos de casamento.

Esses achados suscitam reflexões considerando que o conteúdo dos mitos pode parecer pouco contemporâneo em um contexto de relações amorosas em constantes transformações. Sabe-se que esses mitos vão sendo transmitidos através das gerações pela família, escola, meios de comunicação, etc. É frequente nos dias de hoje a discussão acerca da fragilidade das relações amorosas, da baixa estabilidade das uniões e do pouco compromisso com a conjugalidade. Os participantes deste estudo parecem que estão menos permeáveis a este discurso, pois acreditam na importância do casamento e o vivenciam com relativa estabilidade. A partir destes resultados, abre-se uma agenda para futuros estudos, tais como a de investigar adultos jovens, com menos tempo de união. Investigar sujeitos mais jovens pode ajudar a compreender melhor esse panorama, bem como explorar que outros mitos e crenças a respeito da conjugalidade estão surgindo e influenciando nas relações das novas gerações.

Como limitações deste estudo, considera-se a análise não diádica dos dados, a qual poderia conferir maior especificidade aos resultados, permitindo identificar se há correspondência na adesão aos mitos entre os membros do mesmo casal. Essa perspectiva poderia agregar a percepção individual de cada membro do casal, fornecendo uma compreensão sistêmica do fenômeno.

\section{DECLARAÇÃO DE CONFLITOS DE INTERESSE}

Todas as autoras são coautoras do programa "Viver a dois: compartilhando este desafio", referenciado no estudo. As autoras declaram não possuir conflitos de interesse em relação ao objeto de estudo deste manuscrito.

\section{REFERÊNCIAS}

Andolfi, M. (2018). A terapia familiar multigeracional: Instrumentos e recursos do terapeuta. Belo Horizonte: Artesã.

Andolfi, M., \& Angelo, C. (1989). Tempo e mito em terapia familiar. Porto Alegre: Artes Médicas.

Caldwell, B. E., \& Woolley, S. R. (2008). Marriage and family therapists' endorsement of myths about marriage. The American Journal of Family Therapy, 36(5), 367-387. doi: 10.1080/01926180701804626

Casad, B. J., Salazar, M. M., \& Macina, V. (2015). The real versus the ideal: Predicting relationship satisfaction and well-being from endorsement of marriage myths and benevolent sexism. Psychology of Women Quarterly, 39(1), 119-129. doi: 10.1177/0361684314528304 
Cohen, J. (1988). Statistical power analysis for the behavioral sciences. (2nd ed.). Hillsdale, Erlbaum, 490p.

Costa, C. B. da, Cenci, C. M. B., \& Mosmann, C. P. (2016). Conflito conjugal e estratégias de resolução: Uma revisão sistemática da literatura. Temas em Psicologia, 24(1), 325-338. doi: 10.1590/1982-3703000622016.

Costa, C. B. da, \& Mosmann, C. P. (2015). Relacionamentos conjugais na atualidade: percepções de indivíduos em casamentos de longa duração. Revista da SPAGESP, 16(2), 16-31.

Dancey, C. P., \& Reidy, J. (2006). Estatística sem matemática para psicologia. 3. Ed. Porto Alegre: Artmed.

Delatorre, M. Z., Scheeren, P., \& Wagner, A. (2017). Conflito conjugal: Evidências de validade de uma escala de resolução de conflitos em casais do sul do Brasil. Avances en Psicología Latinoamericana, 35(1), 79-94. doi: 10.12804/revistas.urosario.edu.co/apl/a.3742

Ercegovac, I., \& Bubić, A. (2016). Basic psychological needs predict participants' attitudes and expectations towards marriage. Nordic psychology, 68(2), 7386. doi: $10.1080 / 19012276.2015 .1071200$

Falcke, D. (2003). Águas passadas não movem moinhos? As experiências na família de origem como preditoras da qualidade do relacionamento conjugal. Porto Alegre, RS. Tese de Doutorado. Pontifícia Universidade Católica do Rio Grande do Sul - PUCRS, 185p.

Falcke, D., \& Wagner, A. (2005). A dinâmica familiar e o fenômeno da transgeracionalidade: definição de conceitos. In.: A. Wagner. Como se perpetua a família? A transmissão dos modelos familiares. Porto Alegre: EdiPUCRS.

Fonseca, S. R. A., \& Duarte, C. M. N. (2014). Do namoro ao casamento: Significados, expectativas, conflito e amor. Psicologia: Teoria e Pesquisa, 30(2), 135-143. doi: 10.1590/S0102-37722014000200002.

Fowler, B. J. (2000). Beyond the Myth of Marital Happiness. San Francisco: Jossey-Bass. 243p.

George-Levi, S., Vilchinsky, N., Tolmacz, R., \& Liberman, G. (2014). Testing the concept of relational entitlement in the dyadic context: Further validation and associations with relationship satisfaction. Journal of Family Psychology, 28(2), 193-203. doi:10.1037/a0036150

Galloway, I., Engstrom, E. \& Emmers-Sommer, T. M. (2015). Does movie viewing cultivate young people's unrealistic expectations about love and marriage? Marriage \& Family Review, 51(8), 687-712. doi: 10.1080/01494929.2015.1061629

Honeycutt, J. M. (1991). The endorsement of myths about marriage as a function of gender, age, religious denomination, and educational level. Communication Research Reports, 8(2), 101-111. doi: $10.1080 / 08824099109359881$ 
Jackl, J. A. (2016). "Love doesn't just happen...": Parent-child communication about marriage. Communication Quarterly, 64(2), 193-209. doi: 10.1080/01463373.2015.1103284

Kemer, G., Yıldız, E. Ç., \& Bulgan, G. (2016). Emotional dependency and dysfunctional relationship beliefs as predictors of married Turkish individuals' relationship satisfaction. The Spanish Journal of Psychology, 19(e72), 1-8. doi: 10.1017/sjp.2016.78

Kramer, D., \& Moore, R. M. (2001). Family myths in romantic fiction. Psychological Reports, 88, 29-41. doi: 10.2466/PR0.88.1.29-41

Kurdek, L. A. (1994). Conflict resolution styles in gay, lesbian, heterosexual nonparent, and heterosexual parent couples. Journal of Marriage and the Family, 56(3), 705-722. doi: 10.2307/352880

Lavner, J. A., Karney, B. R., \& Bradbury, T. N. (2013). Newlyweds' optimistic forecasts of their marriage: For better or for worse? Journal of Family Psychology, 27(4), 531-540. doi:10.1037/a0033423

Lazarus, A. (1992). Mitos conjugais. Campinas: Editorial Psy.

Mosmann, C., \& Falcke, D. (2011). Conflitos conjugais: motivos e frequência. Revista da SPAGESP, 12(2), 5-16. doi:

Mosmann, C., Wagner, A., \& Féres-Carneiro, T. (2006). Qualidade conjugal: mapeando conceitos. Paidéia (Ribeirão Preto), 16(35), 315-325. doi: 10.1590/S0103-863X2006000300003.

Neff, L. A., \& Geers, A. L. (2013). Optimistic expectations in early marriage: A resource or vulnerability for adaptive relationship functioning? Journal of Personality and Social Psychology, 105(1), 38-60. doi:10.1037/a0032600

Neumann, A. P. (2017). "Viver a dois: Compartilhando esse desafio": Avaliação de um programa psicoeducativo para casais. Tese de Doutorado. Programa de Pós-Graduação em Psicologia da Universidade Federal do Rio Grande do Sul, Porto Alegre, RS.

Neumann, A. P., \& Wagner, A. (2018). Perspectivas e desafios da coordenação de um programa de educação conjugal. Psico, 49(4), 410-421. doi: 10.15448/1980-8623.2018.4.29350

Rizzon, A. L. C., Mosmann, C. P., \& Wagner, A. (2013). A qualidade conjugal e os elementos do amor: Um estudo correlacional. Contextos Clínicos, 6(1), 4149. doi: $10.4013 /$ ctc. 2013.61 .05

Rust, J., Bennun, I., Crowe, M., \& Golombok, S. (1988). The Golombok Rust Inventory of Marital State. Windsor, NFER-NELSON, 39p.

Scheeren, P., Vieira, R. V. de A., Goulart, V. R., \& Wagner, A. (2014). Marital quality and attachment: The mediator role of conflict resolution styles. Paidéia, 24(58), 177-186. doi: 10.1590/1982-43272458201405

Wagner, A., \& Mosmann, C. (2012). Intervenção na conjugalidade: Estratégias de resolução de conflitos conjugais. In M. N. Baptista \& M. L. M. Teodoro (Eds.), Psicologia de Família (pp. 240-248). Porto Alegre: Artmed. 
Wagner, A., Neumann, A. P., Mosmann, A. P., Levandowski, D. C., Falcke, D., Zordan, E. P., \& Scheeren, P. (2015). 'Viver a dois: Compartilhando esse desafio': Uma proposta psicoeducativa para casais. Porto Alegre: Sinodal.

\section{Sobre as autoras}

Angélica Paula Neumann é psicóloga pela Universidade Regional Integrada do Alto Uruguai e das Missões - URI - Campus de Erechim, Mestre e Doutora em Psicologia pela Universidade Federal do Rio Grande do Sul. Trabalha como Professora no Curso de Psicologia da URI - Campus de Erechim. E-mail: angelicaneumann@gmail.com.

Denise Falcke é psicóloga pela Pontifícia Universidade Católica do Rio Grande do Sul (PUCRS), Mestre e Doutora em Psicologia pela mesma Universidade. Trabalha no Programa de Pós-Graduação em Psicologia da Universidade do Vale do Rio dos Sinos (UNISINOS). Recebe financiamento de pesquisa do CNPq. Email: dfalcke@unisinos.br.

Clarisse Pereira Mosmann é psicóloga pela Pontifícia Universidade Católica do Rio Grande do Sul (PUCRS) e Doutora em Psicologia pela mesma Universidade. Trabalha no Programa de Pós-Graduação em Psicologia da Universidade do Vale do Rio dos Sinos (UNISINOS). Recebe financiamento de pesquisa do CNPq. Email: clarissepm@unisinos.br.

Adriana Wagner é psicóloga pela Pontifícia Universidade Católica do Rio Grande do Sul (PUCRS), Mestre pelo Instituto de Ciencias del Hombre e Doutora em Psicologia pela Universidad Autónoma de Madrid. Trabalha no Instituto de Psicologia da UFRGS e no PPG em Psicologia, sendo bolsista PQ1B do CNPq. Email: adrianaxwagner@gmail.com.

A.W. foi responsável pela obtenção de financiamento, planejamento e supervisão no decorrer do desenvolvimento do projeto, assim como pela revisão final do manuscrito. A.P.N. foi responsável pela coleta e análise dos dados, bem como pela redação inicial (rascunho) e revisão final do artigo. D.F. e C.M. e foram responsáveis pela redação e desenvolvimento do manuscrito. Todas as autoras leram e concordaram com a última versão do manuscrito.

As autoras agradecem ao MCTI e CNPq (Edital Universal MCTI/CNPq no 14/2014) pelo financiamento da pesquisa, assim como à CAPES pela concessão de bolsa de Doutorado à A.P.N. para realização do estudo. Agradecem, ademais, aos profissionais dos centros que colaboraram com a realização do programa Viver a Dois e às bolsistas de iniciação científica e de apoio técnico pelo apoio na coleta de dados. 
Aceito em: 18/12/2019 